\title{
The effect of task structure on diffusion dynamics: Implications for diffusion curve and network-based analyses
}

\author{
WILl HopPITT \\ St Andrews University, St Andrews, Scotland \\ AnNe Kandler \\ University College London, London, England \\ JEREMY R. KENDAL \\ University of Durham, Durham, England \\ AND \\ Kevin N. LaLAND \\ St Andrews University, St Andrews, Scotland
}

\begin{abstract}
Theoretical analyses within the broad field of social learning research give mixed conclusions on whether the shape of a diffusion curve can be used to infer that a learned trait increases through social or asocial learning. Here we explore how factors such as task structure (e.g., multiple-step tasks), task abandonment, subgoal learning, and neophobia affect the shape of the diffusion curve for both asocially learned and socially learned behavior. We demonstrate that, whereas social learning increases the likelihood of S-shaped curves, sigmoidal patterns can be generated by entirely asocial processes, and cannot be reliably interpreted as indicators of social learning. Our findings reinforce the view that diffusion curve analysis is not a reliable way of detecting social transmission. We also draw attention to the fact that task structure can similarly confound interpretation of network-based diffusion analyses, and suggest resolutions to this problem. Supplemental materials for this article may be downloaded from http://lb.psychonomic-journals.org/content/supplemental.
\end{abstract}

There are countless reports of innovations and novel behavior patterns spreading through both human and animal populations (Laland \& Galef, 2009; Lefebvre 1995a, 1995b; Reader, 2004; Rogers, 1995; Zentall \& Galef, 1988). The mathematical function describing the cumulative number of individuals that have acquired a learned trait plotted against time is known as a diffusion curve. In recent years, researchers have expressed differing opinions on whether the characteristic shape of a diffusion curve is potentially a reliable diagnostic of the form of learning underlying the spread of a learned trait. Rogers demonstrated that many thousands of cases of the spread of innovations through human populations exhibit a sigmoidal, or S-shaped, cumulative adoption pattern. In the human innovation literature, such curves are generally assumed to be the product of social learning processes, since individual adopters copy the behavior of others (Rogers, 1995). In such cases, an S-shape is often thought to result because the probability of adoption is a function of the number of both naive and informed individuals (Boyd \& Richerson, 1985; Henrich, 2001). Using mathemati- cal models, Henrich argued that asocial (environmental) learning alone never produces S-shaped curves, and therefore that S-shaped dynamics must imply that social learning, based on biased cultural transmission, underlies the spread of the trait. This reasoning suggests that researchers only require a record of the cumulative number of individuals to have acquired a behavioral trait over time, in order to infer the presence or absence of social learning. Such data have the advantage that they are relatively easy to collect, as evidenced by numerous diffusion studies of this kind on humans and animals (see Rogers, 1995, and Reader, 2004, respectively, for reviews).

In contrast, the animal social learning literature is more equivocal on this issue. Although theoretical models of animal learning also predict S-shaped curves for socially transmitted information and $\mathrm{r}$-shaped decelerating curves for asocially learned traits (Laland, Richerson, \& Boyd, 1993, 1996; Lefebvre, 1995a; Roper, 1986), various researchers have doubted whether such patterns are reliably diagnostic. Roper (1986), Galef (1990), and Rendell and Whitehead (2001) all assumed that an accelerating curve

W. Hoppitt, wjeh1@st-andrews.ac.uk 
can only result from social learning processes, and Lefebvre (1995a) considered this possibility. However, other researchers have questioned, or cautioned against casual interpretation of, this claim (Laland \& Kendal, 2003; Lefebvre, 1995a; Reader, 2004), suggesting that variation among asocial learners, or population structure, could generate accelerating functions through asocial processes alone.

Lefebvre (1995a, p. 236) suggested that "trial-and-error learning could be characterized by a cumulative S-shaped curve at the population level if individual variation in learning latency were normally distributed," giving the false impression that social interaction underpins the diffusion. J. R. Kendal (2002) and Reader (2004) suggested that directed social learning can result in a step-shaped function, with acceleratory component parts. For instance, a novel behavior may spread more rapidly within than between family groups (Fritz, Bisenberger, \& Kotrschal, 2000) and appear to generate step-like cumulative curves. Furthermore, Laland and Kendal (2003) and Reader (2004) argued that, under other circumstances, social learning might lead to curves that either are, or may appear to be, deceleratingfor instance, where researchers fail to detect rare cases early in the diffusion.

Currently, formal analyses of the effect of variation in performance and population structure are lacking, leading to uncertainty as to the validity and seriousness of the aforementioned concerns. Conceivably, social learning processes may typically generate S-shaped curves, and asocial learning processes lead to r-shaped curves, in spite of the impact of these factors, which would lead to the conclusion that diffusion curve analyses have been prematurely dismissed. Conversely, these concerns may be validated by formal theory, in which case some interpretations common in the human innovation literature would be challenged (e.g., Henrich, 2001). Moreover, such validation would naturally lead researchers to ask whether the concerns apply equally to other diagnostic tools currently being used to detect social learning using diffusion data. One newly developed approach is network-based diffusion analysis (NBDA; Franz \& Nunn, 2009, 2010; Hoppitt, Boogert, \& Laland, 2010), which endeavors to isolate social learning by monitoring whether information flows along pathways of association in a social network.

Below, we develop simple statistical models to address these issues. We begin by deploying a continuous time Markov chain model to investigate the shape of the cumulative adoption curve for a task that requires asocial learners to progress through $n$ linear steps to reach the solution, and where individuals vary according to their performance. We go on to consider the effects of individuals abandoning the task - for instance, if they find the task too difficult, or are displaced by other individuals - as well as the impact of reinforcement for learning subgoals, and the effect of neophobia diminishing with time. We then move on to consider the same factors among individuals who acquire the task through social learning. We end by discussing the likely significance of these factors for diffusion curve and NBDA.

\section{THE EFFECT OF TASK STRUCTURE ON THE DIFFUSION CURVE WITH ASOCIAL LEARNING}

Where there are a number of steps required to solve a task, the cumulative diffusion curve may be S-shaped, even if individuals learn the tasks asocially (J. R. Kendal, 2002). For example, an extractive foraging task might require removal of tree bark, followed by digging into the tree cortex, to retrieve insect prey. Similarly, in humans, the manufacture of a composite tool might require learning separately to manufacture the component parts. Here, we investigate more fully the effect that task structure can have on the diffusion curve in the absence of social learning.

\section{Linear $n$-Step Task}

We define a linear $n$-step task to be one in which the learner moves progressively from one state to another, starting in state 0 , and finishing in state $n$, the latter corresponding to solution of the task (see Figure 1A). Transitions between states could represent movements in the environment (e.g., moving to the task), manipulations of the environment (e.g., removal of tree bark), or changes in an individual's internal state (e.g., overcoming neophobia). We model a linear $n$-step task as a continuous time Markov chain, where an individual moves from state $k-1$ to $k$ at rate $\lambda_{k}$. Under purely asocial learning, individuals solve the task independently of one another, and so the expected diffusion curve is the cumulative density function (henceforth CDF) for the time (T) taken to reach state $n$.

We begin with the special case in which $n=1$ (a task requiring only one step to solution), which allows us to assess the effect of variation in the rate of learning. Appendix 1 (in the supplemental materials) establishes that the CDFs are $r$-shaped, and that the magnitude of the variance in asocial learning ability has little impact on the shape of this curve. To some extent, this alleviates Lefebvre's (1995a) concern that variation in asocial learning ability might generate an S-shaped cumulative curve. However, matters become more complex for $n>1$ (see Appendix 2, supplemental materials). The general pattern is that as the number of steps in the task increases, the curve becomes more and more S-shaped. This effect is most pronounced when the steps occur at an equal rate $(\lambda)$, in which case the CDF for $T$ follows that of the gamma distribution with rate parameter $\lambda$ and shape parameter $n$. Conversely, where one step $(D)$ dominates the process $\left(\lambda_{D}<<\lambda_{k \neq D}\right)$, the curve becomes more r-shaped, with the CDF for $T$ converging on an exponential distribution with rate parameter $\lambda_{D}$ (see Figure $2 \mathrm{~A}$ ).

In summary, a linear $n$-step task will result in an S-shaped diffusion curve as a result of entirely asocial learning, unless the rate of learning each step is highly asymmetric.

\section{Resetting $n$-Step Task}

We now consider tasks that can "reset" to their initial state (0) at any stage of the diffusion process (see Figure 1B); we term this a resetting $n$-step task. Resetting could occur if individuals leave the task, either because they "give up" or are displaced by another individual. This 

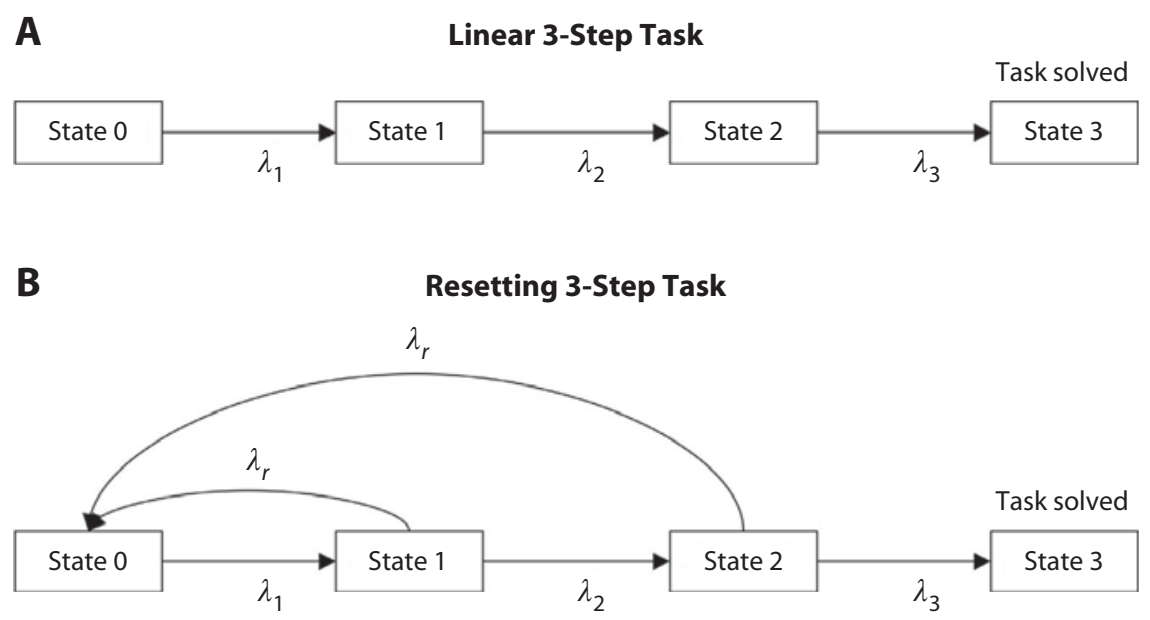

\begin{abstract}
Figure 1. Examples of Markov chains used to model $n$-step tasks. (A) A linear 3-step task. (B) A resetting 3-step task. Arrows represent transitions between states, with $\lambda_{k}(k=1,2, \ldots$, $n-1, n)$ denoting the rate of transition from state $k-1$ to state $k$, and $\lambda_{r}$ denoting the resetting rate, which is the rate of transition from state $i$ to state 0 , where $i \neq 0$.
\end{abstract}

could cause resetting if the task is likely to be lost (e.g., a prey item runs away, a fruit is stolen by another individual), meaning an individual has to find a new task item, or if the nature of the task means it switches back to its initial state when left. If the task can usually be returned to in the same state it was left in, the linear $n$-step task model remains appropriate. Another way in which resetting could occur is if an incorrect manipulation resulted in the task object being ruined (e.g., a tool is damaged during its manufacture; Holzhaider, Hunt, \& Gray, 2010), meaning that the individual has to seek a new task object in its initial state.

Here, we model a resetting $n$-step task as a continuoustime Markov chain similar to that used for the linear case, with an extra set of transitions from states $0>k>n$ to state $k=0$, which occur at rate $\lambda_{r}$ (see Figure 1B). This model assumes that the rate at which individuals move to a state, $k$, is not influenced if they have already reached state $k$ at a previous time in the diffusion. This assumes that no learning occurs before an individual's first final solution of the task, which is consistent with the idea that learning only occurs through direct reinforcement occurring through solution of the task. Later, we go on to consider how other learning processes might alter the process. The analytical derivation of the results for the resetting $n$-step task are given in Appendix 3 (supplemental materials). The general pattern is that, as the rate of resetting, $\lambda_{r}$, increases, the diffusion curve becomes more r-shaped, with the CDF for $T$ converging on that of the exponential distribution (see Figure 2B). Note that the linear $n$-step task is a special case of the more general, resetting $n$-step task with $\lambda_{r}=0$.

This analysis suggests that the expected shape of a diffusion curve resulting from asocial learning is highly influenced by the dynamics of the task-solving process, which might depend not only on the nature of the task itself but also on the context in which it is solved (e.g., the likelihood of being displaced by a competitor, as seen in the study of Lemur catta by R. L. Kendal et al., 2010).

\section{The Effects of Subgoal Learning}

If we assume that learning occurs only through direct reinforcement, and that reinforcement occurs only when the task is solved, the resetting $n$-step task described above is an appropriate model for the time to first solve the task by asocial learning. However, individuals might be sensitive to reaching subgoals, and are intrinsically reinforced for doing so. This would mean that reaching state $k$ increases the future rate at which an individual moves from state $k-1$ to $k\left(\lambda_{k}\right)$. To model this, we assume that learning occurs by a Rescorla-Wagner (1972) learning rule, where a transition to state $k$ changes the future transition rate $\lambda_{k}$, as

$$
\lambda_{k}=\alpha\left(\lambda_{\max , k}-\lambda_{k}\right),
$$

where $\lambda_{\max , k}$ is the maximum transition rate $\lambda_{k}$ for fully "informed" individuals, and $\alpha$ determines the rate at which learning occurs.

Since this model is difficult to solve analytically, we used simulations to investigate the effects of subgoal learning on diffusion curves. We took $n=4, \lambda_{k}(0)=1$, $\lambda_{r}=4$, and varied the value of $\alpha$ from $0,0.1,0.2, \ldots, 1$ and $\lambda_{\max , k}$ as $1,4,10$, and 20. Simulations proceeded as follows.

1. Set $k=0, \lambda_{1}=\lambda_{2}=\lambda_{3}=\lambda_{4}=1$, and $t=0$.

2. Simulate two random numbers, $P$ from an exponential distribution with rate $\lambda_{k+1}$ and $R$ from an exponential distribution with rate $\lambda_{r}=1$.

3. If $P \leq R$, increment $t$ by $P, k$ by 1 , and then $\lambda_{k}$ by $\alpha\left(\lambda_{\max , k}-\lambda_{k}\right)$.

4. If $P>R$, increment $t$ by $R$ and set $k=0$.

5. If $k=4$, stop and store the value of $t$, else return to Step 2.

We ran 10,000 simulations for each combination of parameter values. The results (see Figure 3 ) reveal that as the rate of subgoal learning, $\alpha$, and the resetting rate, $\lambda_{\max , k}$, 


\section{A}

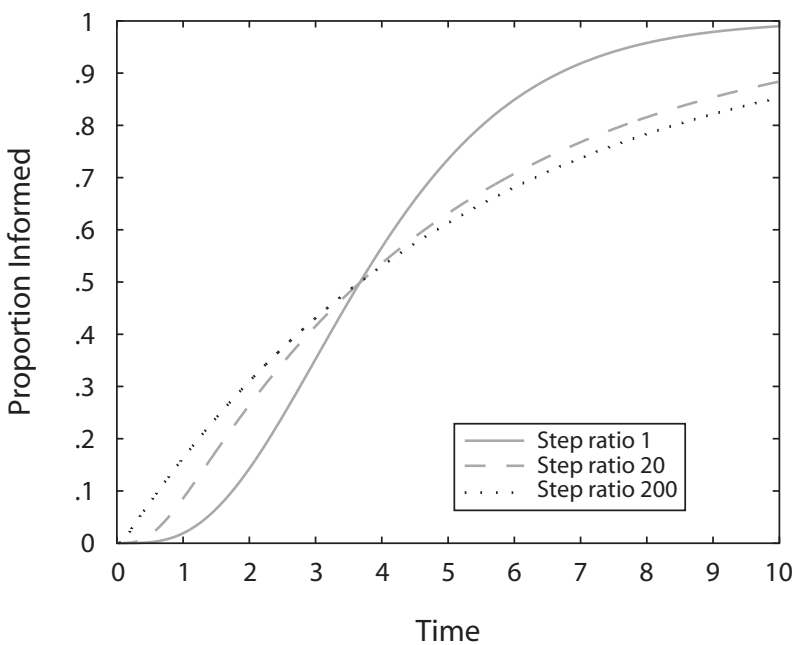

B

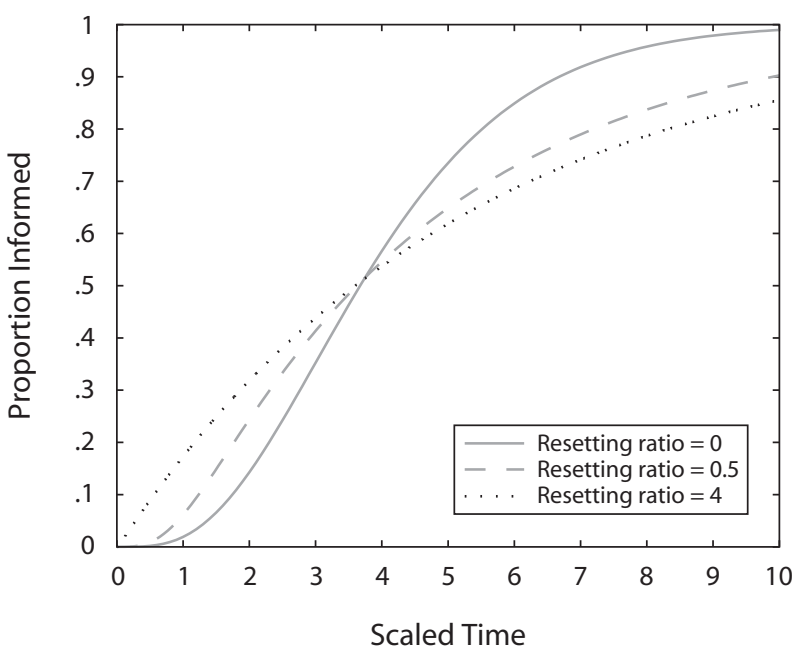

Figure 2. Analytical diffusion curves for asocial learning of a 4-step task. (A) Linear 4-step task, showing the effects of a single step, which we denote $D$, increasingly dominating the process. The step ratio gives the ratio of the expected time to complete step D to the expected time to complete one of the other steps (fixed to be equal). (B) Resetting 4-step task, showing the effects of increasing the resetting rate. All $\lambda_{k}$ are taken to be equal, with resetting ratio $=\lambda_{r} / \lambda_{k}$. For both panels $A$ and $B$, time is scaled such that the proportion informed $=.5$ at time $=4$.

increase, the diffusion curve becomes more S-shaped, becoming more similar to the diffusion curve for a linear 4-step task. Indeed, the curve will be identical to the linear 4-step task when $\alpha=1$ and $\lambda_{\max , k}=\infty$. The analysis indicates that even when we know the resetting rate is high for a task, we might still get an S-shaped diffusion curve through asocial learning.

\section{Reduction in Neophobia}

Another asocial process that might plausibly influence the shape of the diffusion curve is a reduction in neophobia over time, causing individuals to interact with a novel task more, increasing some or all of the transition rates $\lambda_{k}(t)$ between task steps. Here we consider a simple 1-step task in which the rate of transition from naive to informed is a function of time, $\lambda_{1}(t)$. We can derive the CDF for the process by solving the equation

$$
\lambda_{1}(t)=\frac{F^{\prime}(t)}{1-F(t)}
$$

where $F(t)$ is the $\mathrm{CDF}$ for the time to solve, $T$, and $F(0)=0$ (Cox \& Oakes, 1984). We first consider a linear increase in the transition rate

$$
\lambda_{1}(t)=\lambda_{\text {initial }}+\gamma t,
$$

where $\lambda_{\text {initial }}$ is the initial transition rate and $\gamma$ is the rate at which it increases over time (see Figure 4A). This results in the CDF

$$
F(t)=1-\exp \left(-\lambda_{\text {initial }} t-\gamma t^{2}\right)
$$

(see Figure 4B). Alternatively, the effects of neophobia could die away to a baseline rate of transition, for which an exponential model seems appropriate:

$$
\lambda_{1}(t)=\lambda_{\text {baseline }}-\omega \exp (-\rho t),
$$

where $\lambda_{\text {baseline }}$ is the baseline transition rate in the absence of neophobia, $\omega$ is the reduction in rate due to neophobia at $t=0$, and $\rho$ determines the rate at which neophobia dies away (see Figure 4A). This results in the CDF

$$
F(t)=1-\exp -\lambda_{\text {baseline }} t-\frac{\omega}{\rho} \exp (-\rho t)+\frac{\omega}{\rho}
$$

(see Figure 4B). As can be seen in Figure 4, both approaches to modeling neophobia result in an S-shaped diffusion curve.

\section{THE INFLUENCE OF SOCIAL LEARNING ON DIFFUSION CURVES}

We now consider how factors such as task structure affect the diffusion curve for a socially transmitted trait. We model social learning as operating on a varying number of steps, which we feel is likely to reflect the diversity of social learning mechanisms in nature. For example, local enhancement operates only to attract an individual to the task, but observers learn the task by asocial means once they are there. This is likely to result in an increase in rate for only one step. In contrast, some processes, such as production imitation (see Byrne, 2002), result in learning of the entire motor pattern necessary to solve the task. This might result in a simultaneous increase in all, most, or just the later steps associated with a task. We also envisage that in reality a number of "simple" social processes might operate in concert to influence an intermediate number of steps. We assumed that, for each step affected, the rate of transition was a linear function of the number of demonstrators in the population; that is,

$$
\lambda_{k}(t)=1+s d(t)
$$

where $d(t)$ is the proportion of demonstrators at time $t$, and $s$ is the additive effect each additional demonstrator has on the rate of transition to Step $k$. 

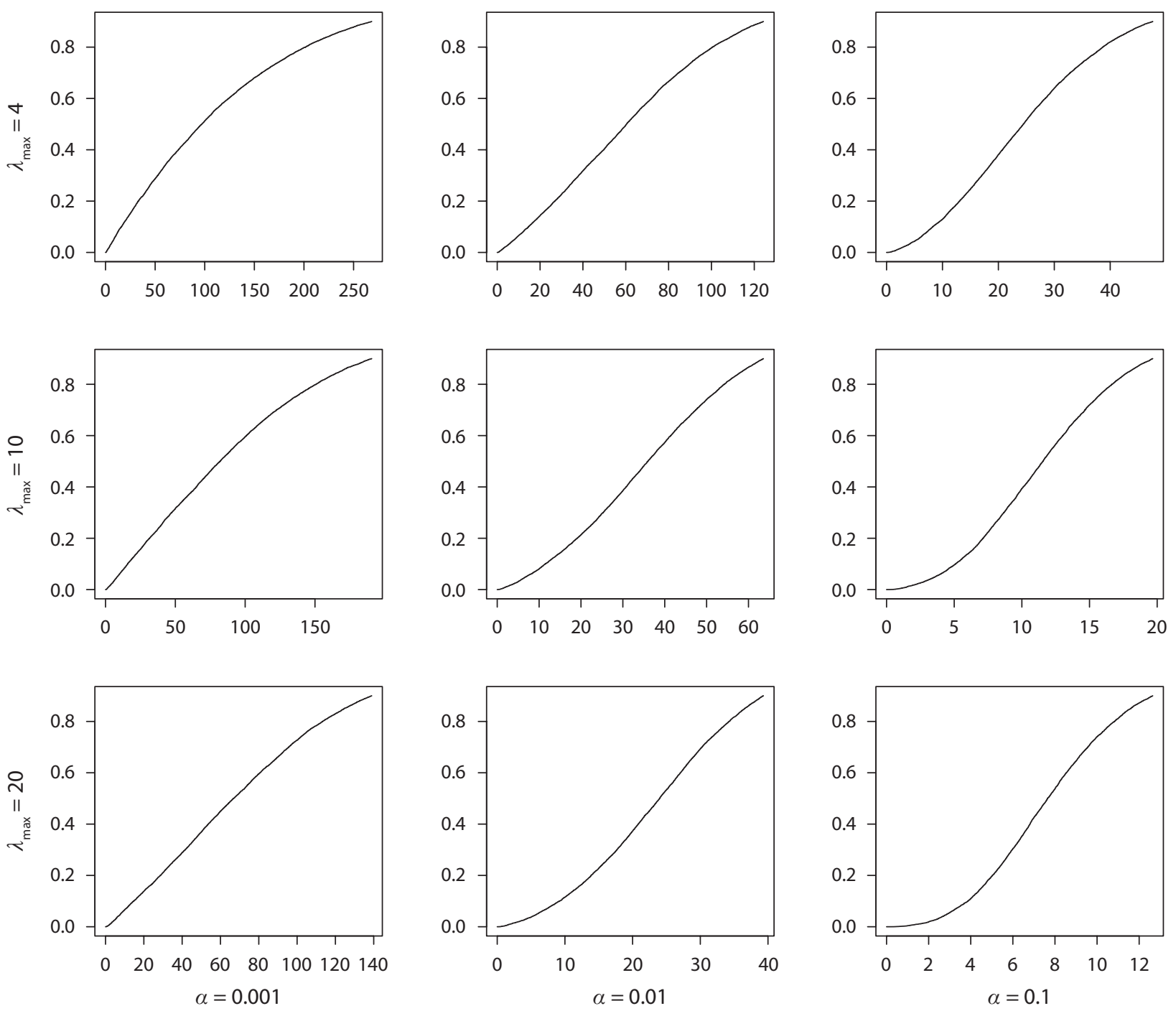

Figure 3. Results of simulations investigating the effect of subgoal learning on the shape of the asocial diffusion curve for a resetting 4-step task. The maximum effect of subgoal learning $\left(\lambda_{\max }\right)$ increases down the $y$-axis, and the rate at which learning occurs $(\alpha)$ increases along the $x$-axis. See the main text for details of the simulations.

To investigate the influence of social learning on the diffusion curves, we used simulations, since in most cases analytical results are difficult to obtain. We simulated a fixed population size of 100 individuals for tasks of $n$ steps with a resetting rate of $\lambda_{r}$. To do this, we used the following procedure.

1. Create a vector, $\mathbf{z}$, of length 100 with all elements equal to 0 . This represents the state of each individual in the population with regard to the task.

2. Set $\lambda_{k}=1$ for $k=1,2, \ldots, n$.

3. For each individual, $i$, draw two random numbers, $P[i]$ from an exponential distribution with rate $\lambda_{z[i]+1}$, and $R[i]$ from an exponential distribution with rate $\lambda_{r}$.

4. If $\min \{P\} \leq \min \{R\}$, increment $t$ by $\min \{P\}$ and increment $z[j]$ by 1 , where $P[j]=\min \{P\}$.
5. If $\min \{P\}>\min \{R\}$ increment $t$ by $\min \{R\}$ and set $z[j]=0$, where $R[j]=\min \{R\}$.

6 . For all $z[i]=n$, record $t$ as the time of solving for individual $i$ and remove from the simulation.

7. Update each $\lambda_{k}$ according to the social learning process being modeled (see below). Return to Step 3.

We ran the model for a 1-step task, a linear 4-step task $\left(\lambda_{r}=0\right)$, and a resetting 4-step task $\left(\lambda_{r}=4\right)$. We found that in all cases, regardless of which social learning process was modeled, social learning caused the diffusion curve to become more S-shaped, as a result of the effect of an increasing number of demonstrators throughout the diffusion. Although the curves became more S-shaped as $s$ increased, the strongest effect on shape was the number of steps on which social learning operated (see Figure 5). 


\section{A}

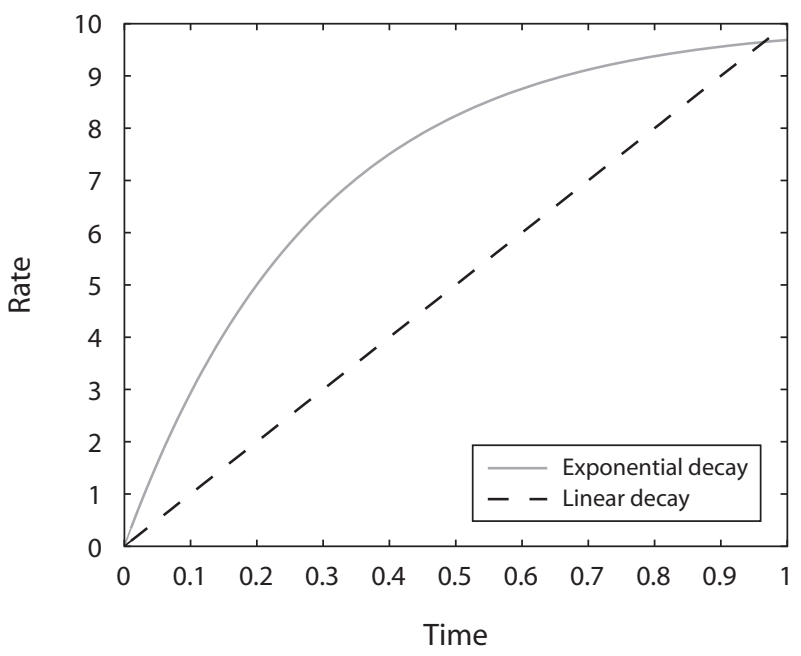

B

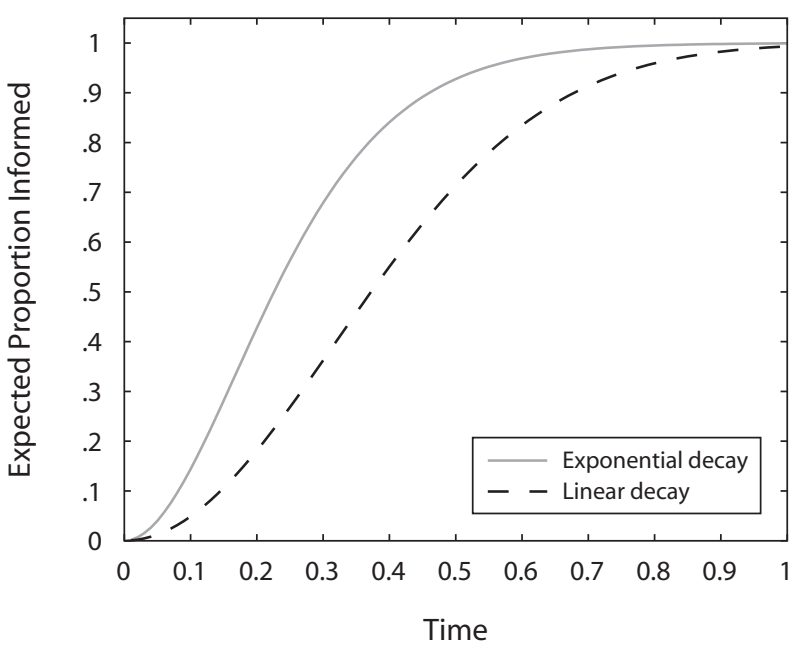

Figure 4. Analytical results for models of the influence of neophobia on a 1-step task. (A) The rate of task solution changes over time for each model. (B) The resulting diffusion curve for each model of neophobia.

\section{Social Learning of Subgoals}

Another mechanism postulated to account for cases of social learning is emulation, whereby observers attempt to re-create the results of the demonstrator's behavior (Tomasello, 1990). One way in which emulation might work is by allowing observers to recognize subgoals which they would otherwise not be able to recognize when they achieve them, thus reinforcing the behavior leading to that subgoal (note that, as with asocial subgoal learning, this process could operate only on a resetting task). We simulated this process as described above, but we also tracked each individual, $i$ 's, rate of transition to state $k, \lambda_{k, i}$. We incremented this when individual $i$ reached state $k$ at time $t$, according to

$$
\lambda_{k, i}=\alpha(t)\left(\lambda_{\max }-\lambda_{k, i}\right)
$$

where $\alpha(t)=v d(t)$ and $0 \leq v \leq 1$. This means that an individual's ability to recognize a subgoal was a linear function of the number of demonstrators in the population.

\section{A}

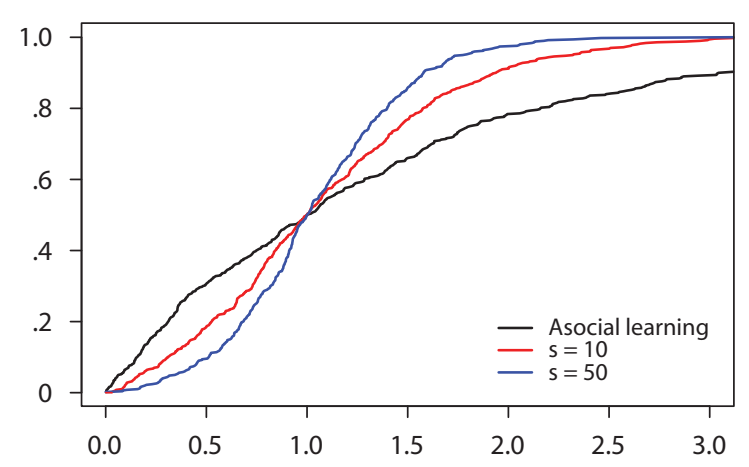

B

Linear 4-Step Task

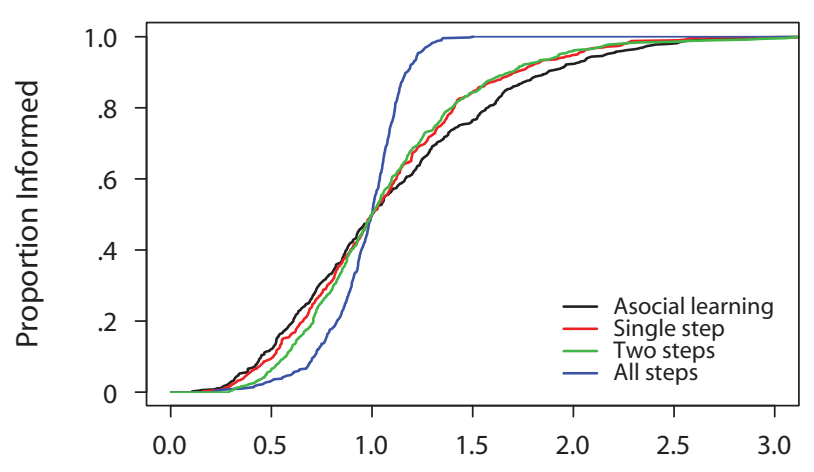

C Resetting 4-Step Task

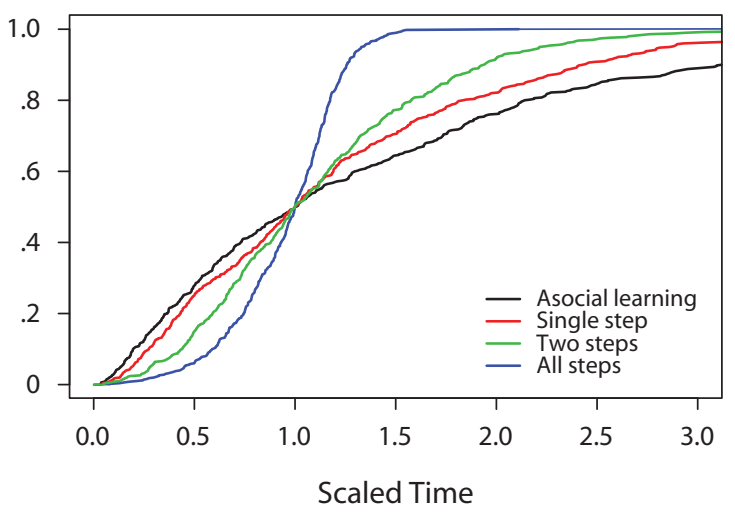

Figure 5. Results of simulations investigating the effects of social learning on the diffusion curves for $\boldsymbol{n}$-step tasks. (A) A 1-step task, showing the effects of different strengths of social learning. (B) A linear 4-step task, showing the effects of social learning when it operates on a differing number of steps $(s=10)$. (C) A resetting 4-step task, showing the effects of social learning when it operates on a differing number of steps $(s=10)$. Simulations are for single populations of 1,000 individuals, and diffusion curves were scaled such that the proportion informed $=.5$ at time $=1$. Social learning is modeled according to Equation 7. 


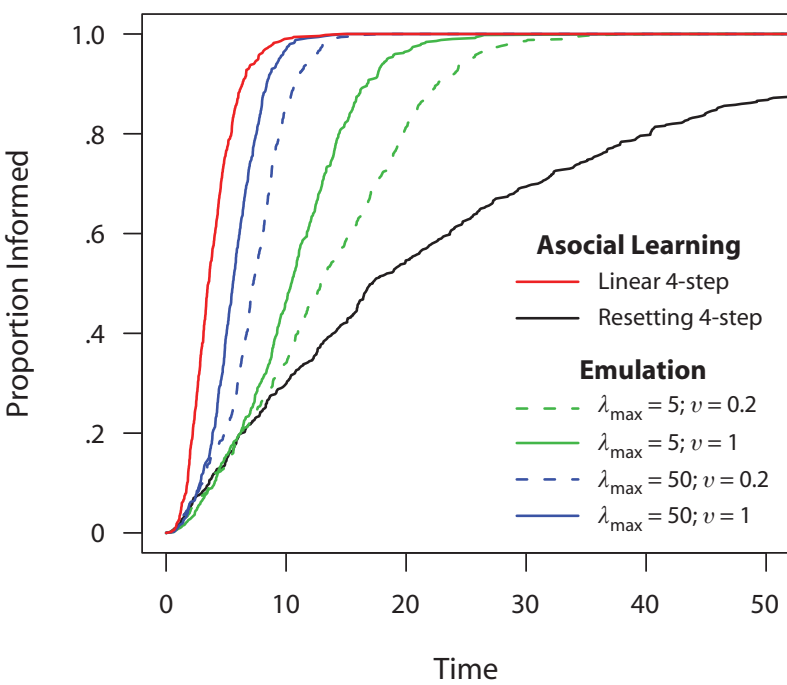

Figure 6. Results of simulations investigating the effects of emulation on the diffusion curve for a resetting 4-step task. Simulated asocial diffusion curves for resetting and linear 4-step tasks are shown for comparison. See main text for the meaning of parameters. Simulations are for populations of 1,000 individuals. Emulation is modeled according to Equation 7.

We found that, as with other types of social learning, emulation made the curve for a resetting task more S-shaped (see Figure 6). Here, the equivalent linear $n$-step task (with $\lambda_{r}=0$ ) sets an upper limit on how S-shaped the curve could be, corresponding to $\lambda_{\max }=\infty$ and $v=1$. This indicates that the change in curvature due to emulation might reflect the number of subgoals the observer is able to learn from the demonstrators' behavior.

\section{IMPLICATIONS FOR DIFFUSION CURVE ANALYSIS}

Taken together, our results strongly suggest that, even with prior knowledge of the nature of the task or innovation, it would be hard for researchers to infer whether a diffusion curve resulted from asocial or social processes. Even a 1-step task acquired through asocial learning, which our analysis suggests would otherwise exhibit an r-shaped cumulative diffusion curve, might have an S-shaped curve if there is a reduction in neophobia of the task over time, or some other process with an equivalent effect. Likewise, a frequently resetting $n$-step task will also have an S-shaped asocial diffusion curve, if attaining subgoals reinforces each individual's behavior.

Social learning can influence the shape of a diffusion curve, typically generating or enhancing S-shaped patterns. However, a social influence will remain difficult to detect in a single diffusion curve unless researchers know what the expected diffusion curve would be under asocial learning alone. In principle, researchers could make the case that a specific task has a relatively high rate of resetting, and that, consequently, the asocial diffusion curve would be r-shaped, or might attempt to quantify the maximum number of steps involved in the task as a conserva- tive null hypothesis. Unfortunately, our analysis suggests that such attempts are likely to be misleading, since processes such as neophobia and subgoal reinforcement are likely to make the diffusion curve more S-shaped, resulting in false positive reports of social learning.

In our analysis, we suggest that different social learning mechanisms are likely to affect diffusion dynamics in different ways, by altering the different rates of transition within the task or by allowing an individual to learn about task subgoals. However, we find that the overall effect of different psychological mechanisms on the diffusion curve is always qualitatively similar: All social learning processes make the diffusion curve more S-shaped. Therefore, we suggest that it is unlikely that social learning mechanism can be inferred from a diffusion curve alone, even if a researcher were able to uncover the shape of the diffusion curve in purely asocial conditions. However, our models suggest that one might study social learning mechanism from detailed diffusion data in which each individual's movements between states (e.g., naive to knowledgeable/ at proximity or distance from a task) is tracked, and modeled as a function of the presence of demonstrators (e.g., J. R. Kendal, R. L. Kendal, \& Laland, 2007).

Our findings reinforce the view that diffusion curve analysis is not a reliable way of detecting social transmission (Reader, 2004), and raise questions about whether the S-shaped curves reported for the spread of human innovations (Rogers, 1995) can legitimately be viewed as resulting from biased cultural transmission (Henrich, 2001). The models presented here make the assumption that populations are "well-mixed," in that all individuals are equally likely to interact with each other; this assumption is also made by standard diffusion curve analysis. ${ }^{1}$ Other analyses suggest that violations of this assumption can also result in inflated Type I errors for social learning and call for the need for methods that take this heterogeneity into account (Franz \& Nunn, 2009). Our analyses suggest that even if the "well-mixed" assumption is upheld, the use of the shape of diffusion curves to draw inferences about the underlying learning processes is unreliable, and we caution against this practice. In our judgment, alternative methods for detecting social learning, such as the option-bias method (R. L. Kendal et al., 2010; R. L. Kendal, J. R. Kendal, Hoppitt, \& Laland, 2009), analyses of the relationship between behavioral similarity and measures of association (Matthews, 2009; Whitehead, 2009), analyses of the spatial spread of a trait (Lefebvre, 1995b), and NBDA (Franz \& Nunn, 2009, 2010; Hoppitt et al., 2010), offer greater promise (see Kendal, Galef, \& van Schaik, 2010, for a summary of methods). However, our analysis also suggests that other methods of analyzing diffusion data, such as NBDA, might also be vulnerable to Type I errors, unless one takes the possible effects of task structure, neophobia, and the like into account, an issue to which we now turn.

\section{IMPLICATIONS FOR NBDA}

Alternative methods are beginning to emerge for detecting social transmission using diffusion data and are re- 
viewed in this collection. These methods include NBDA, a method initially developed by Franz and Nunn (2009) and extended by Hoppitt et al. (2010). NBDA infers social transmission if the spread of a diffusion follows the pattern of association between individuals, known as the social network. Franz and Nunn's method takes as data the times at which individuals first acquire the behavioral trait in question, and fits a model of social learning that assumes that the rate of transmission of behavior between two individuals is proportional to the rate of association between them.

Hoppitt et al. (2010) introduced a variant of NBDA that makes the same assumptions about social transmission but uses only the order in which individuals acquire the behavioral trait. Hoppitt et al. called their version "order of acquisition diffusion analysis" (OADA) and contrasted it with Franz and Nunn's "time of acquisition diffusion analysis" (TADA). Both methods fit a model that includes social learning, and compare it with a model with only asocial learning, inferring social learning if the former provides a better fit to the data. Hoppitt et al. found that TADA had better power to detect an effect when the assumptions of the model were upheld, but that it was vulnerable to false positives if there was a systematic increase in the baseline rate of acquisition, absent social transmission. In contrast, OADA was not vulnerable to false positives under these conditions; the reason for this can be understood by comparing both models in a common framework. Both approaches can fit a model including both asocial and social learning of the form

$$
\lambda_{i}(t)=\lambda_{0}(t)\left[1-z_{i}(t)\right]\left[s \sum_{j=1}^{N} a_{i, j} z_{j}(t)+(1-s)\right],
$$

where $\lambda_{i}(t)$ is the rate at which individual $i$ acquires the trait; $\lambda_{0}(t)$ is the baseline rate of acquisition, in the absence of social transmission; $z_{i}(t)$ gives the status of individual $i$ $(1=$ informed; $0=$ naive $) ; s$ is a fitted parameter estimating the relative strength of social transmission, ranging from 0 (all learning asocial) to 1 (all learning is social transmission); and $\alpha_{i, j}$ is the association or network connection between individuals $i$ and $j$, reflecting the rate of social transmission from $j$ to $i .^{2}$ The model is fitted to the data using maximum likelihood, and social transmission is inferred if a model with $s>0$ is significantly better than a model with $s=0$. The key difference between the two methods is that TADA requires that the baseline rate of acquisition $\lambda_{0}(t)$ be specified or fitted to the data, whereas OADA merely assumes the baseline rate to be the same for all individuals. The current versions of TADA (Franz \& Nunn, 2009; Hoppitt et al., 2010) assume that the baseline rate is constant: $\lambda_{0}(t)=\lambda_{0}$. In the context of this article, this assumption corresponds to a 1-step task, or approximately to a resetting $n$-step task with a high rate of resetting without processes such as neophobia or subgoal learning. This means that TADA is vulnerable in the same circumstances described above as diffusion curve analysis (see simulations in Appendix 5, supplemental materials).

In Appendix 4 (supplemental materials), we modify TADA so that an inhomogeneous baseline rate function, $\lambda_{0}(t)$, can be fitted to the data, allowing one to control for such effects in detecting social transmission. Our analysis above indicates that the obvious candidate is the gamma distribution, for which the CDF becomes S-shaped as the shape parameter increases. We would expect the gamma distribution to be exact for a linear $n$-step task, with equal step transition rates, in the absence of neophobia, and so on. Therefore, instead of fitting a single parameter for the constant baseline rate, we fit two parameters determining the shape of the baseline rate function. We find that this modified version of TADA has an appropriate Type I error rate and is still able to detect social learning from data simulated from our $n$-step task model (see Appendix 5, supplemental materials).

\section{CONCLUSION}

The primary function of the mathematical models presented here was to assess whether the shape of the diffusion curve can be used as a diagnostic tool for detecting social learning, as has often been assumed in both the human and animal literature (see, e.g., Rogers, 1995; Roper, 1986). We conclude that, even if the assumption of a well-mixed population with no spatial heterogeneity in resources is accepted, the use of diffusion curves remains problematic, since S-shaped curves can arise through a number of plausible asocial processes. We also conclude that alternative methods for detecting social learning from diffusion data, such as NBDA, need to be formulated in a way that controls for this effect.

\section{AUTHOR NOTE}

W.H. was supported by BBSRC Grant BB/D015812/1, A.K. by a Leverhulme Trust Early Career Fellowship, J.R.K. by an RCUK Research Fellowship, and K.N.L. by BBSRC Grants BB/C005430/1 and BB/ D015812/1. Correspondence concerning this article should be addressed to W. Hoppitt, School of Biology, St Andrews University, St Andrews KY16 9TS, Scotland (e-mail: wjeh1@st-andrews.ac.uk).

\section{REFERENCES}

Boyd, R., \& Richerson, P. J. (1985). Culture and the evolutionary process. Chicago: University of Chicago Press.

Byrne, R. W. (2002). Imitation of novel complex actions: What does the evidence from animals mean? In P. J. B. Slater, J. S. Rosenblatt, C. T. Snowdon, \& T. J. Roper (Eds.), Advances in the study of behavior (Vol. 31, pp. 77-105). San Diego: Academic Press.

Cox, D. R., \& OAKes, D. (1984). Analysis of survival data. London: Chapman \& Hall.

Franz, M., \& NunN, C. L. (2009). Network-based diffusion analysis: A new method for detecting social learning. Proceedings of the Royal Society B, 276, 1829-1836.

Franz, M., \& NunN, C. L. (2010). Investigating the impact of observation errors on the statistical performance of network-based diffusion analysis. Learning \& Behavior, 38, 235-242.

Fritz, J., Bisenberger, A., \& Kotrschal, K. (2000). Stimulus enhancement in greylag geese: Socially mediated learning of an operant task. Animal Behaviour, 59, 1119-1125.

Galef, B. G., JR. (1990). Tradition in animals: Field observations and laboratory analyses. In M. Bekoff \& D. Jamieson (Eds.), Readings in animal cognition (pp. 91-105). Cambridge, MA: MIT Press.

HENRICH, J. (2001). Cultural transmission and the diffusion of innovations: Adoption dynamics indicate that biased cultural transmission is the predominate force in behavioral change and much of sociocultural evolution. American Anthropologist, 103, 992-1013. 
Holzhaider, J. C., Hunt, G. R., \& Gray, R. D. (2010). Social learning in New Caledonian crows. Learning \& Behavior, 38, 206-219.

Hoppitt, W., Boogert, N. J., \& Laland, K. N. (2010). Detecting social transmission in networks. Journal of Theoretical Biology, 263, 544555. doi:10.1016/j.jtbi.2010.01.004

KeNDAL, J. R. (2002). An investigation into social learning: Mechanisms, diffusion dynamics, functions and evolutionary consequences. Unpublished doctoral thesis, University of Cambridge, Cambridge, England.

Kendal, J. R., Kendal, R. L., \& Laland, K. N. (2007). Quantifying and modelling social learning processes in monkey populations. International Journal of Psychology \& Psychological Therapy, 7, 123-138.

Kendal, R. L., Custance, D. M., Kendal, J. R., Vale, G., Stoinski, T. S., Rakotomalala, N. L., \& Rasamimanana, H. (2010). Evidence for social learning in wild lemurs (Lemur catta). Learning \& Behavior, 38, 220-234.

Kendal, R. L., Galef, B. G., JR., \& van Schaik, C. P. (2010). Social learning research outside the laboratory: How and why? Learning \& Behavior, 38, 187-194.

Kendal, R. L., Kendal, J. R., Hoppitt, W., \& Laland, K. N. (2009). Identifying social learning in animal populations: A new "option-bias" method. PLOS ONE, 4, e6541. doi:10.1371/journal.pone.0006541

Laland, K. N., \& Galef, B. G., JR. (EDs.) (2009). The question of animal culture. Cambridge, MA: Harvard University Press.

Laland, K. N., \& KendaL, J. R. (2003). What the models say about social learning. In D. Fragaszy \& S. Perry (Eds.), The biology of traditions: Models and evidence (pp. 33-55). Cambridge: Cambridge University Press.

LALAND, K. N., Richerson, P. J., \& Boyd, R. (1993). Animal social learning: Toward a new theoretical approach. In Perspectives in ethology: Behavior and evolution (Vol. 10, pp. 249-277). New York: Plenum.

Laland, K. N., Richerson, P. J., \& Boyd, R. (1996). Developing a theory of animal social learning. In C. M. Heyes \& B. G. Galef, Jr. (Eds.), Social learning in animals: The roots of culture (pp. 129-154). San Diego: Academic Press.

Lefebvre, L. (1995a). Culturally-transmitted feeding behaviour in primates: Evidence for accelerating learning rates. Primates, 36, 227239 .

LEFEBVRE, L. (1995b). The opening of milk bottles by birds: Evidence for accelerating learning rates, but against the wave-of-advance model of cultural transmission. Behavioural Processes, 34, 43-53.

Matthews, L. J. (2009). Intragroup behavioral variation in whitefronted capuchin monkeys (Cebus albifrons): Mixed evidence for social learning inferred from new and established analytical methods. Behaviour, 146, 295-324.

READER, S. M. (2004). Distinguishing social and asocial learning using diffusion dynamics. Learning \& Behavior, 32, 90-104
ReADER, S. M., \& LALAND, K. N. (2000). Diffusion of foraging innovation in the guppy. Animal Behaviour, 60, 175-180.

Rendell, L. E., \& Whitehead, H. (2001). Culture in whales and dolphins. Behavioral \& Brain Sciences, 24, 309-382.

Rescorla, R. A., \& Wagner, A. R. (1972). A theory of Pavlovian conditioning: Variations in the effectiveness of reinforcement and nonreinforcement. In A. H. Black \& W. F. Prokasy, Classical conditioning II: Current research and theory (pp. 64-99). New York: Appleton-Century-Crofts.

Rogers, E. (1995). Diffusion of innovations (4th ed.). New York: Free Press.

ROPER, T. J. (1986). Cultural evolution of feeding behaviour in animals. Science Progress, 70, 571-583.

Tomasello, M. (1990). Cultural transmission in the tool use and communicatory signalling of chimpanzees? In S. T. Parker \& K. R. Gibson (Eds.), "Language" and intelligence in monkeys and apes: Comparative developmental perspectives (pp. 274-311). New York: Cambridge University Press.

WhiteheAd, H. (2009). How might we study culture? A perspective from the ocean. In K. N. Laland \& B. G. Galef, Jr. (Eds.), The question of animal culture. Cambridge, MA: Harvard University Press.

YouNG, H. P. (2008). Innovation diffusion in heterogeneous populations: Contagion, social influence, and social learning. Available at www economics.ox.ac.uk/Research/wp/pdf/paper303.pdf.

Zentall, T. R., \& Galef, B. G., JR. (Eds.) (1988). Social learning. Psychological and biological perspectives. Hillsdale, NJ: Erlbaum.

\section{NOTES}

1. In some cases, heterogeneity has been incorporated on the basis of the assumption that individuals differ in their propensity to adopt a novel trait (Rogers, 1995; Young, 2008), but the population is still assumed to be "well mixed."

2. Hoppitt et al. (2010) also allowed inclusion of variables that model differences in asocial learning rate between individuals. Here, these are excluded for clarity. Franz and Nunn (2009) used a different parameterization; here, we use Hoppitt et al.'s (2010) notation to facilitate comparison of TADA and OADA.

\section{SUPPLEMENTAL MATERIALS}

Five appendixes containing additional analyses may be downloaded from http://lb.psychonomic-journals.org/content/supplemental.

(Manuscript received March 11, 2010; accepted for publication April 28, 2010. 